\title{
Substances toxiques persistantes (PTS) dans les corps gras : État des lieux d'après le rapport du programme des Nations-Unies pour I'environnement (PNUE)
}

Oléagineux, Corps Gras, Lipides. Volume 10, Numéro 4, 265-73, JUILLET-AOÛT 2003, Problématiques actuelles dans le domaine de l'analyse des oléagineux et des corps gras

Auteur(s) : Jean François NARBONNE, Pr. de Toxicologie, LPTC, UMR 5472, CNRS-Université Bordeaux 1351 cours de la Libération 33405 Talence.

Author(s) : Jean François NARBONNE

Résumé : Depuis plus de trente ans, une attention croissante a été portée sur les risques pour la santé humaine posés par la contamination de l'environnement par les substances toxiques persistantes (PTS). Ces composés persistent pendant de longues périodes dans les compartiments abiotiques et biotiques, sont transportés sur de longues distances depuis leurs points sources et s'accumulent chez l'homme et les organismes via les chaînes alimentaires. En 1997, le PNUE propose une liste initiale de 12 substances appelées polluants organiques persistants (POPs): aldrine, endrine, dieldrine, chordane, DDT, heptachlore, toxaphène, mirex, hexachlorobenzène, PCBs, dioxines (PCDDs), furanes (PCDFs). En 2001, de nouveaux composés sont ajoutés sur la liste des PTS en fonction de leurs caractéristiques comme la liposolubilité, leur résistance à la dégradation, leur forte toxicité ou la constance des apports: hexachlorohexane, PAHs, endosulfan, pentachlorophénol, formes organiques des métaux lourds: mercure, étain et plomb, phtalates, PBDEs\\PBBs, chlordécone, alkyl phénols, atrazine. Les sources de PTS sont diverses : (1) Utilisation de produits chimiques industriels comme les pesticides ou les emballages, (2) Réservoirs historiques déjà présents dans l'environnement essentiellement dans les sols et les sédiments ou sous forme de stocks périmés, (3) Sous-produits de process, en particulier comme contaminants indésirables de procédés industriels et de combustion. La France a été associée au rapport de la région IV méditerranéenne. Les niveaux dans les compartiments de l'environnement sont rapportés et l'exposition de I'homme via I'alimentation (vecteur essentiel) est estimée en fonction des données collectées. Une évaluation des risques associés a été faite quand les données étaient suffisantes (PAHs, PCBs, PCDD \F, Me Hg). Les lipides apparaissent comme vecteurs majeurs des PTS à travers la chaîne alimentaire. Toutefois, les données sur les niveaux de contamination et leur évolution dans le temps sont souvent absentes pour certains composés et \lou certaines zones comme le Sud et le SudEst du bassin. Une évaluation scientifique des risques associant de façon cohérente la population, les activités, les émissions, les transports et les effets dans un réseau de coopération internationale est fortement souhaitable.

Summary : For more than thirty years awareness has been growing about the dangers for human health and the environment by persistent toxic substances (PTS). These chemicals persist for long periods, travel large distances from their point of origin to the environment and bioaccumulate in humans and other organisms through the food chain. In 1997 UNEP proposed an initial list of twelve chemicals designated as Persistent Organic Pollutants (POPs): Aldrin, Endrin, Dieldrin, Chordane, DDT, Heptachlor, Toxaphene, Mirex, Hexachlorobenzene, PCBs, Dioxins (PCDDs), Furans (PCDFs). In 
2001 additional chemicals were added related to characteristics in lipid solubility, low degradation, high toxicity, constant input: Hexachlorohexane, PAHs, Endosulfan, Pentachlorophenol, Organic forms of Mercury, Tin and Lead, Phtalates, PBDEs\\PBBs, Chlordecone, Alkyl phenols, Atrazine. Sources of PTS are related to (1) release of manufactured products, specially pesticides and materials in contact with food, (2) historical reservoir already existing in the environment, predominantly found in soil, sediments and stockpiles, (3) by products from unintentional generation process, specially unwanted contaminants from industrial or combustion treatments. France was included in the assessment of Mediterranean Region IV. Levels in environmental compartments were reported and human exposure mainly by foods was evaluated by compilation of existing data. Tentative of risk evaluation was performed. Lipids appeared to be the vector of PTS contamination through the food chain. However, information on contaminant levels and trends is still lacking for certain contaminants and media in some areas particularly the South and South-Eastern basin. An integrated science based approach linking population, activities, emissions, transport and effects in a coherent international cooperative risk assessment framework is needed.

Mots-clés : PTS, POPs, accumulation, transport, contamination, risques

Keywords : PTS, POPs, accumulation, transport, contamination, risks

\section{ARTICLE}

\section{Auteur(s) : Jean François NARBONNE}

Pr. de Toxicologie, LPTC, UMR 5472, CNRS-Université Bordeaux 1351 cours de la Libération 33405 Talence

\section{Introduction}

En 1997, le programme des Nations Unies pour l'Environnement (PNUE) décide qu'une action immédiate doit être initiée au niveau international en vue de protéger la santé humaine et l'environnement des effets d'un groupe initial de 12 substances désignées comme polluants organiques persistants (POPs), en particulier en prenant des mesures urgentes de réduction et/ou d'élimination de leurs émissions ou de leurs rejets. Dans ce cadre, des négociations internationales ont été initiées et ont abouti à la signature de la convention de Stockholm en 2001. Cependant il apparaît vite que cette première initiative, limitée aux POPs, mérite d'être étendue à d'autres substances toxiques persistantes (PTS). Une liste de 16 substances est alors ajoutée (tableau 1). Pour établir les priorités d'action, un outil d'évaluation a été constitué sous l'égide du Global Environment Facility (GEF), ayant pour objectif de réunir des données scientifiques aux niveaux national, régional et mondial de façon à définir les mesures de contrôle de réduction ou d'élimination des PTS, d'identifier les domaines d'ignorance et de stimuler en conséquence des recherches complémentaires à initier. Pour réaliser cet énorme travail, une division des tâches par grandes régions du globe a été décidée, dans le but de collecter les données auprès de toutes sources d'information publiques ou privées et de produire une évaluation scientifique des risques induits par ces substances sur l'environnement et la santé humaine. II a donc été décidé de rédiger un rapport pour chacune des douze régions de globe, puis de réaliser un rapport global de synthèse. En fait, 
chacune des 12 régions a été constituée en essayant d'associer des pays développés (ayant des infrastructures administratives de recherche et de surveillance) avec des pays moins développés. II s'agit des régions suivantes : I-Arctique, II-Amérique du Nord, III - Europe, IV - Méditerranée, V - Afrique Sub-Saharienne, VI - Océan Indien, VII - Asie Centrale et du Nord-Est, VIII - Asie du SudEst et Sud Pacifique, IX - lles du Pacifique, X-Amérique Centrale et Caraïbes, XI-Amérique du SudEst et Ouest, XII-Antarctique. La région Méditerranéenne (Région IV) comprend la France, I'Espagne, le Portugal, l'Italie, les pays de l'ancienne Yougoslavie, I'Albanie, la Grèce, la Turquie, la Syrie, le Liban, la Jordanie, Israël, la Palestine, I'Egypte, la Libye, la Tunisie, I'Algérie, le Maroc, Chypre et Malte, l'Andorre, Monaco et San Marin.

Tableau 1. Contribution relatives des différents pays aux stocks de PCBs dans la région méditerranéenne en 1995.

\begin{tabular}{l|l|l|l|}
\hline Stocks de PCBs (tonnes) & \% du stock de la région & \\
\hline Espagne & 5972 & 24 \\
\hline France & 5846 & 12 \\
\hline Italie & 3872 & 12 \\
\hline Algérie & 3055 & 11 \\
\hline Croatie & 2082 & 7 \\
\hline Grèce & 1768 & 8 \\
\hline Tunisie & 2000 & 3 \\
\hline Portugal & 657 & 1 \\
\hline Ancienne & 197 & \\
\hline Yougoslavie & & & \\
\hline
\end{tabular}

\section{Les PTS}

Le terme PTS ou Persistant Toxic Substances ne concerne pas de risques particuliers mais fait référence au temps de persistance dans l'environnement. En effet, leurs caractéristiques physicochimiques rendent ces substances capables d'être bioaccumulées. La plupart de ces composés sont liposolubles et leur persistance est due soit à leur faible dégradabilité, soit à la constance des apports. Les produits chlorés sont particulièrement concernés puisque la présence d'halogènes dans un composé organique diminue sa solubilité dans l'eau, augmente sa solubilité dans les lipides (huiles et graisses) et limite les possibilités de dégradations oxydatives. Ce sont donc des composés très biodisponibles, pouvant être bioaccumulés (concentration dans les graisses entraînant des teneurs supérieures dans les tissus que dans le milieu) et biomagnifiés (augmentation des concentrations dans les chaînes alimentaires). Les caractéristiques générales communes des PTS sont donc: persistance, faible solubilité dans l'eau, forte toxicité, production et utilisation non compatibles avec une économie durable. Le cheminement des PTS se fait donc via les lipides des organismes 
constituant les chaînes alimentaires. La maîtrise des niveaux de contamination des corps gras destinés à l'alimentation humaine ou des animaux domestiques passe donc par la connaissance des sources, flux et transferts de ces composés. Un premier corps de 12 substances a été désigné par le terme "POPs ou polluants organiques persistants » fixés par la convention de Stockholm. Il s'agit des composés suivants: Aldrine, Endrine, Dieldrine, Chordane, DDT, Heptachlore, Toxaphène, Mirex, Hexachlorobenzène, PCBs, Dioxines (PCDDs), Furanes (PCDFs). En fait il existe d'autres substances pouvant être considérées comme persistantes et toxiques et qui ne répondent pas exactement aux caractéristiques des POPs mais posant le même type de problème. Il s'agit des composés suivants : Hexachlorohexane, PAHs, Endosulfan, Pentachlorophénol, formes organiques des métaux lourds: Mercure, Etain et Plomb, Phtalates, PBDEs/PBBs, Chlordécone, Alkyl phenols, Atrazine.

\section{Sources de PTS}

Les substances considérées comme PTS ont plusieurs origines :

1) Il peut s'agir de produits manufacturés pour des utilisations industrielles (par exemple emballages) ou agricoles (une part importante est constituée de pesticides),

2) II peut s'agir de produits qui ont été utilisés dans le passé mais qui subsistent dans des compartiments de l'environnement qui jouent le rôle de réservoirs (principalement le sol et les sédiments) ou existant sous forme de stocks non utilisés mais non détruits,

3) II peut s'agir enfin de sous-produits indésirables formés au cours des procédés industriels ou naturels comme les combustions.

Pour les pays méditerranéens, il est assez difficile d'obtenir des informations officielles sur les émissions de PTS à partir des sources principales comme les industries ou les centres urbains. En fait la plupart des autorités nationales ne les recensent pas systématiquement ni de façon centralisée et les informations contenues dans le rapport ont été obtenues par des "réseaux parallèles ", le plus souvent constitués à partir de contacts personnels des rédacteurs, en particulier dans les pays du Sud. Les informations obtenues peuvent être résumées de la façon suivante :

1) Pour les pesticides persistants (y compris le Lindane), les sources sont multiples et diffuses. Bien que I'on constate des diminutions dans leurs utilisations conformément aux interdictions et limitations d'emploi successives, il y a un manque de données et de contrôle sur les stocks des produits obsolètes.

2) Pour les PTS industriels comme les PCBs, la production des pays européens du Sud (France, Espagne et Italie) a été de l'ordre de 300000 tonnes pour la période 1954-1984. Les stocks répertoriés dans différents pays sont rapportés dans le tableau 1. Les équipements existants contenant des PCBs sont encore mal estimés et la majeure capacité d'élimination de ces produits est située en France. L'ensemble des émissions est en décroissance mais des sites fortement contaminés ont été identifiés, en particulier du fait de la destruction d'installations électriques ou militaires pendant les conflits régionaux comme les guerres des Balkans ou israélo-libanaise. 3) Pour les sous-produits comme les Dioxines et Furans, les sources sont essentiellement thermiques. L'amélioration des procédés et des contrôles dans les usines d'incinération (malheureusement un peu tardive dans certains pays comme la France) a amené une forte diminution des émissions. Les sources secondaires proviennent des industries métallurgiques, de la combustion du bois et de la 
matière végétale. L'évaluation des émissions de dioxines pour différents pays européens de la région IV est présentée dans le tableau 2.

Tableau 2. Evaluation des émissions de dioxines dans les pays européens de la région IV jusqu'à 2005 (en g I-TEQ/an).

\begin{tabular}{|c|c|c|c|c|}
\hline Sources & Données 1995 & Données 2000 & Projection 2005 & \\
\hline \multirow[t]{3}{*}{ France } & $\begin{array}{l}\text { Total des sources } \\
\text { considérées }\end{array}$ & $1350-1529$ & $804-949$ & $692-813$ \\
\hline & Sources industrielles & $987-1027$ & $461-461$ & $340-340$ \\
\hline & Sources non-industrielles & $363-502$ & $343-488$ & $352-473$ \\
\hline \multirow[t]{3}{*}{ Grèce } & $\begin{array}{l}\text { Total des sources } \\
\text { considérées }\end{array}$ & $89-136$ & 90-135 & $91-136$ \\
\hline & Sources industrielles & $55-58$ & $56-56$ & $58-58$ \\
\hline & Sources non-industrielles & $34-79$ & $34-79$ & $34-78$ \\
\hline \multirow[t]{3}{*}{ Italie } & $\begin{array}{l}\text { Total des sources } \\
\text { considérées }\end{array}$ & $366-967$ & $370-985$ & $227-628$ \\
\hline & Sources industrielles & $271-620$ & $281-648$ & $153-303$ \\
\hline & Sources non-industrielles & $95-348$ & $89-336$ & $74-325$ \\
\hline \multirow[t]{3}{*}{ Portugal } & $\begin{array}{l}\text { Total des sources } \\
\text { considérées }\end{array}$ & $90-136$ & $88-133$ & $82-123$ \\
\hline & Sources industrielles & $17-54$ & $17-52$ & $13-45$ \\
\hline & Sources non-industrielles & $73-82$ & $72-81$ & $69-78$ \\
\hline \multirow[t]{3}{*}{ Espagne } & $\begin{array}{l}\text { Total des sources } \\
\text { considérées }\end{array}$ & $131-388$ & $117-327$ & $122-323$ \\
\hline & Sources industrielles & $77-184$ & $64-132$ & $71-137$ \\
\hline & Sources non-industrielles & $54-203$ & 53-195 & $51-187$ \\
\hline
\end{tabular}

4) Pour les autres PTS la situation régionale est la suivante : a. La source principale des PAHs est liée aux opérations de transport du pétrole. En dehors des marées noires, les relargages sont de l'ordre de 1000 tonnes par an alors que les émissions continentales sont de 40-700 tonnes par an et que les apports par les rivières sont estimés à 40 tonnes par an. 
b. Les relargages de peintures antifouling utilisées pour les bateaux de commerce sont la source principale de TBT et sont estimés à 240 tonnes par an. Les principaux ports de commerce sur les côtes nord-ouest de la Méditerranée ou de l'Adriatique sont des "points chauds " potentiels. c. Les émissions de composés polybromés utilisés comme ignifugeants sont proportionnelles à la consommation d'équipements électriques et électroniques. Bien que leur utilisation augmente, les niveaux de contamination apparaissent faibles pour l'instant. d. Les émissions des détergents non ioniques sont très importantes, de l'ordre de 167000 tonnes par an du fait de l'usage massif de lessives ( 6 millions de tonnes par an), les pays de l'UE contribuant pour $50 \%$ à cet apport.

II semble qu'un contrôle sévère des stocks de PCB et de pesticides obsolètes soit une priorité. D'autre part les transports par cargo sont une source majeure d'hydrocarbures et de TBT pour la région, qu'il convient donc de mieux maîtriser.

\section{Accumulation et transport des PTS dans l'environnement}

Les données collectées sont davantage issues d'études ponctuelles que de résultats de réseaux constitués de surveillance. II s'agit donc de données locales ou liées à des sites particulièrement contaminés. Il y a donc de nombreux manques pour dresser un tableau complet de la situation pour les pays riverains de la Méditerranée, en particulier pour des compartiments comme l'atmosphère, l'eau de boisson, les sols, les boues d'épuration, ou pour les PTS d'intérêt plus récent comme les dioxines et les alkyl phénols, en particulier dans les pays du Sud. De plus l'absence de méthodes standardisées rend difficiles les comparaisons entre les sites et dans le temps.

Malgré ces limites, les données compilées montrent que les PTS sont largement distribuées dans l'environnement mais de façon hétérogène. Par exemple, les teneurs en HCB et en $\mathrm{\gamma}$-HCH vont de 2,4 et $1,5 \mathrm{ng} / \mathrm{m}^{3}$ respectivement dans les zones agricoles du Nord de la France, à moins de $0,1 \mathrm{ng} / \mathrm{m}^{3}$ dans des zones éloignées. Les concentrations en DDT dans les sédiments varient dans une fourchette de 0,25 à $885 \mathrm{ng} / \mathrm{g}$ suivant les sites. Pour les PCBs on note des valeurs de 1,3 à $7274 \mathrm{ng} / \mathrm{g}$, les valeurs supérieures correspondant à des sites pollués comme les sources d'émission ou les estuaires de rivières drainant des sites industriels ou urbains. De même les organismes aquatiques, en particulier ceux utilisés comme espèces sentinelles par les réseaux de surveillance, ont des concentrations en PTS liées aux niveaux de pollution de leur habitat. Les valeurs maximales sont observées chez les mammifères marins, avec par exemple des concentrations de $400 \mu \mathrm{g} / \mathrm{g}$ en DDT ou de $1400 \mu \mathrm{g} / \mathrm{g}$ en PCBs dans les graisses cervicales de dauphins de Méditerranée, valeurs supérieures à celle mesurées chez les dauphins de l'Atlantique. On note aussi des valeurs supérieures dans les œufs de mouettes collectés dans la partie Ouest du bassin méditerranéen que dans ceux collectés dans la partie Est. De même les niveaux de pollution sont plus élevés dans le bassin méditerranéen que dans l'Atlantique Nord ou dans l'Arctique. Un exemple d'évolution des concentrations dans un réseau trophique est donné dans le tableau 3, issu des résultats du programme SEINE-AVAL. Pour les PCBs on note une forte biomagnification pour le congénère $C B 153$ qui se retrouvera donc majoritaire dans la graisse de poisson, ce qui n'est pas le cas pour le CB 77 dioxine-like. Pour les PAHs on observe une faible biomagnification pour le Phénanthrène chez le poisson, au contraire on observe une diminution des concentrations en BaP le long de la chaîne, ce qui indique une augmentation des capacités de métabolisation et d'excrétion, en particulier chez les animaux. De même, si on passe au niveau trophique supérieur, c'est-à-dire chez les mammifères marins, on note que la bioaccumulation 
dépend de deux facteurs antagonistes : liposolubilité et capacité d'excrétion. Ainsi on note de forts pouvoirs de biomagnification pour les composés suivants: DDT, PCBs (congénères non coplanaires), Toxaphène, Chlordane, PBDEs, $\beta-\mathrm{HCH}$, Dieldrine, Chloro-paraffines, PBBs. Au contraire on observe des diminutions des concentrations en passant des poissons aux mammifères marins pour les composés suivants : $\mathrm{PCBs}$ (congénères coplanaires $\mathrm{DL}$ ), $\mathrm{\gamma}-\mathrm{HCH}, \mathrm{HCB}$ et certaines dioxines.

Tableau 3. Transferts des PCBs (77 et 153) et des PAHs (Phénathrène et Benzo(a)pyrène) dans une chaîne alimentaire de l'estuaire de la Seine (programme SEINE-AVAL). Les résultats sont exprimés en $n g / g$ matière sèche.

\begin{tabular}{|c|c|c|c|c|}
\hline CB 153 & CB 77 & Phe & BaP & \\
\hline Sédiments & 10 & 0,3 & 125 & 170 \\
\hline Phytoplancton & 15 & 0,2 & 97 & 124 \\
\hline Copépode & 28 & 0,1 & 72 & 4,4 \\
\hline Crustacé & 157 & 0,6 & 11 & 1,8 \\
\hline Poisson & 200 & 0,6 & 235 & 0,2 \\
\hline
\end{tabular}

Les données sur l'évolution des contaminations dans les poissons, les moules ou les œufs d'oiseaux marins au cours des dernières décennies sont particulièrement intéressantes. On observe une diminution générale des teneurs en DDT entre les années soixante et quatre- vingt-dix, ce qui correspond aux mesures réglementaires d'interdiction ou de restriction de production ou d'utilisation. On peut classer les PTS en fonction de leur courbe de décroissance: DDT > HCHs > > PCBs > PAHs. Cependant pour les PCBs et les PAHs la plupart des sites côtiers de la Méditerranée et de l'Adriatique montrent plutôt une stabilité des teneurs et des apports. Des sites particulièrement pollués ont été identifiés, par exemple les estuaires de la Seine, du Rhône, de l'Ebre, du Po et du Nil, les baies ou lagunes de Izmit, d'Iskenderun et Venise, les rivières comme le Po, l'Ebre, la Seine, la Kupa et la Lepenica ou les lieux de décharge à proximité de Durres, Skopje, Alger ou Mostaganem. Certains sites ont été étudiés de façon pluridisciplinaire comme l'estuaire de la Seine, la lagune de Venise et certains sites contaminés au cours de la guerre des Balkans. Les données collectées concernent surtout les PCBs, DDT, HCHs, PAHs, HCB et TBTs. Pour les autres PTS comme les phtalates, les alkylphénols et les PBDE/PBBs, les données sont largement insuffisantes. Les transports à longue distance des PTS se font essentiellement par voies atmosphérique et aquatique. La modélisation des flux aériens intercontinentaux montre un déplacement de l'Europe vers I'Afrique avec une pointe particulière à la saison chaude. On peut estimer que les masses aériennes européennes peuvent atteindre la zone équatoriale en 3-4 jours. En fait s'il apparaît évident que le bassin Ouest de la Méditerranée et l'Adriatique Nord sont des réservoirs et des sources de PTS, les données manquent pour apprécier précisément les zones de retombées sur les pays du Sud. 


\section{Contamination des aliments et risques pour l'homme}

La contamination de l'environnement par les PTS a pour conséquence l'exposition de l'homme, essentiellement par voie alimentaire et une pollution de ses graisses sur de longues périodes (vie entière).

\section{Teneurs dans les tissus}

La démonstration de la contamination humaine a été apportée par la compilation des teneurs en PTS dans les graisses du sang, du tissu adipeux ou du lait. Pour les pesticides organochlorés, des données existent pour certains pays du Sud où l'utilisation a souvent perduré après les interdictions en Europe. Par exemple en Jordanie on note en 1998 des teneurs dans le lait humain de 860, 1400 et 3300 ng/g de matière grasse (MG) pour l'aldrine, la dieldrine et l'endrine, respectivement. En 1997, on rapporte 500 et $190 \mathrm{ng} / \mathrm{g}$ pour l'heptachlore et l'heptochlore-epoxyde, respectivement. Les données sont plus abondantes pour les DDTs. Cependant on note de grandes différences en fonction de l'année de prélèvement et dans la répartition entre $D D T$ et $D D E$. Ainsi les valeurs publiées pour les années 1985-1995 montrent des valeurs de $79 \mathrm{ng} / \mathrm{g}$ poids frais en Israël et de $240 \mathrm{ng} / \mathrm{g}$ en Egypte, dans les régions d'utilisation intense. En Jordanie les valeurs sont passées de 1 060-2 640 ng/g MG en 1993 à 5-18 ng/g en 2000. Des données voisines sont rapportées pour la Turquie et la Grèce. En Espagne, des valeurs de 0,7-1,5 $\mathrm{gg} / \mathrm{g}$ MG et de 3,7-6,3 $\mu \mathrm{g} / \mathrm{g}$ MG ont été rapportées en 1989-1993 pour le DDT et DDE, respectivement. En Italie centrale, les teneurs en DDT dans le tissu adipeux ont diminué de $25 \%$ au cours de la dernière décennie. Pour I'HCB les données sont très hétérogènes (de 0,8 à $4000 \mathrm{ng} / \mathrm{g} \mathrm{MG)}$. Certaines données semblent indiquer une régression des valeurs ou cours de la dernière décennie (Croatie) alors que les données de la Jordanie, de la Turquie et de l'Italie montrent plutôt une certaine stabilité de la contamination avec une corrélation positive avec l'âge des personnes étudiées. Les données sur les $P C B s$ varient aussi dans de grandes proportions du fait des différences dans l'expression des résultats analytiques (somme de tous les congénères, somme des 3 principaux, somme des 6 ou 7 indicateurs, somme des congénères dioxine-like en TEQs....). Les principaux résultats sont résumés dans la figure 1. Il faut aussi remarquer la baisse des teneurs en $P C B$ dans le lait chez la femme en fonction du nombre d'enfants. Pour le congénère $C B 101$, on note une diminution de la concentration dans le lait de $24 \%$ au deuxième enfant et de $36 \%$ pour le troisième enfant.

Les dioxines ont été essentiellement mesurées dans le lait humain comme indiqué dans le tableau 4. II faut noter une diminution des teneurs moyennes depuis les années 1990 de l'ordre de 30 à $40 \%$ en France et en Espagne, et des valeurs plus élevées en Egypte (20,4 pg I-TEQ/g MG) qu'en Israël (10,2 pg l-TEQ/g MG) ou en Palestine (6,46 pg I-TEQ/g MG). Si les mesures dans le lait humain concernent les femmes en âge de procréer (l'âge moyen est d'environ 30 ans), les dosages dans le sang sont plus représentatifs de l'ensemble de la population. Les études chez l'homme (figure 2) montrent une augmentation des teneurs en dioxines des lipides sanguins en fonction de l'âge. La pente moyenne est d'environ 0,55 pg TEQ/g MG/an pour les pays européens et peut varier de 0,565 pg TEQ/g MG/an pour Tarragone et 0,790 pg TEQ/g MG/an à Madrid. Les données collectées pour les teneurs en HCHs dans les tissus humains datent de la période 1983-1997 (figure 3). On note une forte prédominance des teneurs en isomère $\beta$ et des évolutions différentes dans le temps pour I'Espagne (décroissance) et pour la Jordanie (augmentation) pour l'isomère $\gamma$. Une seule étude sur les teneurs en $P B B s$ et $P B D E s$ dans les tissus humains a été réalisée en Espagne et montre des teneurs de 
1 à 2 ng/g MG dans le tissu adipeux pour les principaux congénères. Pour les PAHs, les demi-vies chez I'homme sont très courtes, de l'ordre de quelques heures à quelques jours, du fait de leur fort taux de métabolisation. En fait les risques pour la santé sont plus liés à la constance de l'exposition et non aux risques de bioaccumulation. Il s'agit plus d'évaluer une " dose interne » à l'aide de biomarqueurs qu'une charge corporelle par mesure directe dans les tissus. De plus, il existe de grandes variations individuelles pour les fumeurs ou les résidents à proximité de voies majeures de circulation. Ainsi une étude italienne sur l'excrétion de métabolite $1-\mathrm{OH}$ du pyrène dans I'urine montre une diminution significative entre 1996 et 2000 à la fois chez les fumeurs et chez les non-fumeurs. Les taux d'adduits à l'ADN des lymphocytes du sang périphérique sont de 1,39 à 2,4 adduits $/ 10^{8}$ nucléotides en Grèce et en Italie.

Tableau 4. Niveaux de dioxines dans les tissus humains (pg TEQ/g matière grasse).

\begin{tabular}{|c|c|c|c|c|c|}
\hline $\mathbf{n}$ & M & SD & Fourchette & Références & \\
\hline \multicolumn{6}{|l|}{ France (Lait) } \\
\hline - 1990 (Paris) & 15 & 20,1 & ND & ND & $\begin{array}{l}\text { Gonzales et al. } \\
1996\end{array}$ \\
\hline (9 départements) 1998 & 15 & 21,2 & 2,08 & $14,6-35,2$ & Sokolsky, 1998 \\
\hline (19 départements) 2000 & 244 & 16,5 & 5,07 & $6,5-34,3$ & Fery et al. 2000 \\
\hline (2 départements) 2002 & 6 & 15,8 & 0,7 & $9,7-18,8$ & AFSSA \\
\hline (17 départements) & 17 & 11,8 & 1,58 & $7,2-23,3$ & Sokolsky, 2003 \\
\hline \multicolumn{6}{|l|}{ Egypte (Lait) } \\
\hline - 1997 (4 villes) & $6 / 87$ & 20,4 & ND & $11-24,1$ & Malish et al. 2000 \\
\hline \multicolumn{6}{|l|}{ - Espagne } \\
\hline - 1996 (Tarragona) Lait & 15 & 11,8 & 2,71 & $5,9-17,1$ & $\begin{array}{l}\text { Shumacher et al. } \\
1998\end{array}$ \\
\hline $\begin{array}{l}\text { - } 1996 \text { (Tarragona) } \\
\text { Sang }\end{array}$ & 20 & 27,0 & 8,2 & $14-49$ & $\begin{array}{l}\text { Shamacher et al. } \\
1998\end{array}$ \\
\hline
\end{tabular}

\section{Evaluation des risques}

II s'agit en premier lieu d'évaluer les dangers en répertoriant les effets toxiques potentiels liés aux différentes PTS, en situant les doses induisant ces effets ou reconnues comme sûres (doses 
journalières tolérables ou doses virtuellement sûres pour les composés cancérigènes). Dans un deuxième temps il s'agit d'évaluer les niveaux d'exposition (essentiellement alimentaire) pour l'ensemble, ou des segments de la population. L'évaluation des risques se fait en comparant les niveaux d'exposition aux valeurs toxicologiques de référence (DJT, DVS). Le tableau 5 présente les données toxicologiques liées aux pesticides organochlorés classés comme PTS. Les effets principaux sont neurotoxiques, hépatotoxiques, cancérogenèse, les perturbations hormonales affectant en particulier les fonctions de reproduction ou thyroïdiennes. Les données sur l'exposition alimentaire sont limitées à une étude italienne donnant un apport moyen pour un adulte de $16 \mathrm{ng}$ et $5,3 \mathrm{ng} / \mathrm{kg} /$ jour pour le $D D T$ et les $H C H s$ respectivement, soit $0,08 \%$ et $1,8 \%$ des DJT édictées par I'OMS. Ces données plutôt rassurantes aujourd'hui ne doivent cependant pas exclure des situations locales à risque et il n'y a pas de données sur les effets retardés dus à de fortes expositions passées, c'est-à-dire dans les années 1950 à 1970.

Tableau 5. Propriétés toxicologiques des pesticides organochlorés classés comme PTS.

\begin{tabular}{|c|c|c|c|c|c|c|}
\hline Utilisation & T1/2 sols & $\begin{array}{l}\text { Bioconcentrati } \\
\text { on BCFs }\end{array}$ & $\begin{array}{l}\text { CL50 poisso } \\
\text { ns }\end{array}$ & $\begin{array}{l}\text { DL50 mammifè } \\
\text { re }\end{array}$ & $\begin{array}{l}\text { Effets } \\
\text { toxiques }\end{array}$ & \\
\hline Aldrine & $\begin{array}{l}\text { Insecticide } \\
\text { sur } \\
\text { parasites } \\
\text { des sols et } \\
\text { des bois }\end{array}$ & $20 \mathrm{j}-1,6$ ans & Oui & $2,2-53 \mu \mathrm{g} / \mathrm{l}$ & $\begin{array}{l}33- \\
320 \mathrm{mg} / \mathrm{k} \\
\mathrm{g}\end{array}$ & $\begin{array}{l}\text { Neurotoxique } \\
\text { Hépatotoxique }\end{array}$ \\
\hline Dieldrine & $\begin{array}{l}\text { Sur } \\
\text { insectes du } \\
\text { sol }\end{array}$ & 3-4 ans & Oui & $1,1-4,1 \mathrm{mg} / \mathrm{l}$ & $\begin{array}{l}40- \\
70 \mathrm{mg} / \mathrm{kg}\end{array}$ & $\begin{array}{l}\text { Neurotoxique } \\
\text { Hépatotoxique }\end{array}$ \\
\hline Endrine & $\begin{array}{l}\text { Insecticide } \\
\text { sur } \\
\text { parasites } \\
\text { de grandes } \\
\text { cultures, } \\
\text { rodonticide }\end{array}$ & 5-12 ans & $14-18000$ & $<1 \mu \mathrm{g} / \mathrm{l}$ & $\begin{array}{l}\text { 3- } \\
43 \mathrm{mg} / \mathrm{kg}\end{array}$ & $\begin{array}{l}\text { Neurotoxique } \\
\text { Hépatotoxique }\end{array}$ \\
\hline Chlordane & $\begin{array}{l}\text { Insecticide } \\
\text { sur } \\
\text { parasites } \\
\text { domestiqu } \\
\text { es }\end{array}$ & 4 ans & Oui & $0,4-90 \mathrm{mg} / \mathrm{l}$ & $\begin{array}{l}200- \\
590 \mathrm{mg} / \mathrm{k} \\
\mathrm{g}\end{array}$ & $\begin{array}{l}\text { Perturbateur } \\
\text { endocrinien } \\
\text { Cancérogène } \\
\text { possible }\end{array}$ \\
\hline $\begin{array}{l}\text { Heptachlo } \\
\text { re }\end{array}$ & $\begin{array}{l}\text { Insectes du } \\
\text { sol, du } \\
\text { coton, } \\
\text { moustique }\end{array}$ & $0,75-2$ ans & Oui & $<0,11 \mu \mathrm{g} / \mathrm{kg}$ & $\begin{array}{l}40- \\
119 \mathrm{mg} / \mathrm{k} \\
\mathrm{g}\end{array}$ & $\begin{array}{l}\text { Cancérogène } \\
\text { discuté }\end{array}$ \\
\hline
\end{tabular}




\begin{tabular}{|c|c|c|c|c|c|c|}
\hline & s (malaria) & & & & & \\
\hline DDT & $\begin{array}{l}\text { Insecticide } \\
\text { (malaria, } \\
\text { typhus) }\end{array}$ & $<15$ ans & $\begin{array}{l}50000 \\
\text { (poisson) } \\
500000 \\
\text { (bivalves) }\end{array}$ & $1,5-56 \mathrm{mg} / \mathrm{l}$ & $\begin{array}{l}118 \mathrm{mg} / \mathrm{k} \\
\mathrm{g}\end{array}$ & $\begin{array}{l}\text { Estrogénomimétiq } \\
\text { ue Cancérogène } \\
\text { possible }\end{array}$ \\
\hline Toxaphène & $\begin{array}{l}\text { Insecticide } \\
\text { acaricide }\end{array}$ & 100 j à 12 ans & $\begin{array}{l}4247- \\
51400 \\
\text { (poissons) }\end{array}$ & 1,8 à $22 \mu \mathrm{g} / \mathrm{l}$ & $\begin{array}{l}49- \\
365 \mathrm{mg} / \mathrm{k} \\
\mathrm{g}\end{array}$ & $\begin{array}{l}\text { Perturbateur } \\
\text { endocrinien } \\
\text { Cancérigène } \\
\text { potentiel }\end{array}$ \\
\hline Mirex & $\begin{array}{l}\text { Insecticide } \\
\text { sur fourmis } \\
\text { et additif } \\
\text { matériaux }\end{array}$ & $<10$ ans & $\begin{array}{l}2600- \\
51400 \\
\text { (poissons) }\end{array}$ & $0,2-30 \mathrm{mg} / \mathrm{l}$ & $\begin{array}{l}235 \mathrm{mg} / \mathrm{k} \\
\mathrm{g}\end{array}$ & $\begin{array}{l}\text { Perturbateur } \\
\text { endocrinien } \\
\text { Cancérigène } \\
\text { potentiel }\end{array}$ \\
\hline HCB & $\begin{array}{l}\text { Fongicide } \\
\text { sur } \\
\text { semence }\end{array}$ & $2,7-5,7$ ans & Oui & $50-200 \mu \mathrm{g} / \mathrm{l}$ & $\begin{array}{l}0,25 \mathrm{mg} / \mathrm{k} \\
\mathrm{g}\end{array}$ & $\begin{array}{l}\text { Hépatotoxique, } \\
\text { cancérigène } \\
\text { possible }\end{array}$ \\
\hline $\mathrm{HCHs}$ & $\begin{array}{l}\text { Insecticide } \\
\text { sur sols et } \\
\text { semences } \\
\text { biocide } \\
\text { ménager }\end{array}$ & $1-2$ ans & Faible & $20-90 \mathrm{~g} / \mathrm{l}$ & $\begin{array}{l}60- \\
250 \mathrm{mg} / \mathrm{k} \\
\mathrm{g}\end{array}$ & $\begin{array}{l}\text { Perturbateur } \\
\text { endocrinien } \\
\text { mutagène }\end{array}$ \\
\hline $\begin{array}{l}\text { Endosulfa } \\
\mathrm{n}\end{array}$ & $\begin{array}{l}\text { Insecticide } \\
\text { acaricide, } \\
\text { traitement } \\
\text { des bois }\end{array}$ & $35-150 \mathrm{j}$ & Non & $1,5 \mu \mathrm{g} / \mathrm{l}$ & $\begin{array}{l}18- \\
160 \mathrm{mg} / \mathrm{k} \\
\mathrm{g}\end{array}$ & $\begin{array}{l}\text { Perturbateur } \\
\text { endocrinien }\end{array}$ \\
\hline PCP & $\begin{array}{l}\text { Insecticide } \\
\text { fongicide } \\
\text { traitement } \\
\text { des bois }\end{array}$ & $23-178 j$ & Non & $0,093-0,5 \mathrm{mg} / \mathrm{l}$ & $\begin{array}{l}50- \\
210 \mathrm{mg} / \mathrm{k} \\
\mathrm{g}\end{array}$ & $\begin{array}{l}\text { Perturbateur } \\
\text { endocrinien }\end{array}$ \\
\hline
\end{tabular}

Le tableau 6 résume un certain nombre de données sur les PTS autres que les pesticides organochlorés. Pour les PAHs l'étude la plus complète est celle réalisée par I'AFSSA montrant une exposition alimentaire moyenne pour un adulte de 8 à $10 \mu \mathrm{g}$ TEQ (équivalent $\mathrm{BaP}$ ) $/ \mathrm{kg} / \mathrm{j}$ pour les 6 PAHs cancérigènes, avec comme vecteur majeur les viandes cuites (de 62 à $76 \%$ ) et un risque évalué par comparaison avec les DVS calculées par le RIVM hollandais correspondant à 2 cancers supplémentaires par million d'habitants pour une exposition vie entière. Pour les dioxines, le niveau moyen d'exposition alimentaire pour un adulte est estimé de 1,06 à 1,6 pg TEQ/ $/ \mathrm{kg} / \mathrm{j}$, avec une contribution majeure des produits animaux dont le lait (30-25\%) et les produits de la mer (28-33\%). Ceci représente 100 à $160 \%$ de la DJT donnée pour les dioxines (1 pg TEO/kg/j). II faut noter les 
conséquences significatives de la fermeture des incinérateurs polluants en France sur la qualité du lait distribué sur le marché (figure 4). Cependant certains consommateurs vivant à proximité de sources importantes d'émission (en particulier les " petits " incinérateurs en zone rurale ou les forts consommateurs de certains poissons peuvent dépasser largement la DJT de 3 à 4 fois et il existe toujours de grandes incertitudes sur les seuils d'apparition d'effets toxiques comme les cancers, le diabète ou les dérèglements hormonaux. De même, pour ces catégories de consommateurs, la contamination des graisses corporelles peut entraîner des niveaux élevés d'exposition pour le fœtus (via le sang du cordon) et pour le nouveau-né (via le lait) avec la possibilité d'effets neurologiques et hormonaux. Pour les $P C B$ s il existe deux approches d'évaluation des risques suivant que l'on fait référence aux PCBs indicateurs ou aux PCBs D-L. Dans chaque cas on peut se référer à la DJT définie pour les substances dioxine-like (2,33 pg TEQ/ kg/j soit 1,33 pg TEQ pouvant être affectée aux PCB-DL, ou à la DJT récemment définie par l'OMS pour l'ensemble des congénères $(20 \mathrm{ng} / \mathrm{kg} / \mathrm{j})$. Le vecteur alimentaire principal est constitué par les poissons et produits de la mer (75\%), les niveaux d'exposition étant estimés à $2,5 \mathrm{pg}$ TEQ $/ \mathrm{kg} / \mathrm{j}$ pour les PCB-DL (soit $187 \%$ de la DJT d'après une étude de I'UE) et à $36,8 \mathrm{ng} / \mathrm{kg} / \mathrm{j}$ pour les $7 \mathrm{PCBs}$ indicateurs (soit $184 \%$ de la DJT, d'après une étude italienne). On constate donc que l'évaluation des risques est identique quand le calcul concerne I'ensemble des PCBs ou simplement la fraction D-L. Ces dépassements de DJA restent inquiétants du fait de la possibilité d'exposition des fœtus et des nouveau-nés via les lipides du sang et du lait de la mère. Pour les composés polybromés (BFRs) il y peu de données pour les pays de la région IV. D'après des données européennes on peut évaluer une exposition moyenne à $7 \mathrm{ng} / \mathrm{kg} / \mathrm{j}$ avec comme vecteur majeur les produits de la mer. II n'existe pas de DJT pour ces composés, mais étant donné la similitude des mécanismes d'action de ces composés avec les PCBs, on peut se référer par défaut à la DJT donnée pour les PCBs. Cette exposition alimentaire estimée correspond dans ce cas à $30 \%$ de la DJT, ce qui peut devenir préoccupant étant donné la forte utilisation actuelle de ces composés, laissant prévoir une augmentation des contaminations et la constitution de réservoirs. Pour les autres composés comme les alkyl phénols et les phtalates, les données sont très insuffisantes pour servir de base à un calcul d'exposition et à une évaluation des risques, ce qui est problématique pour des substances utilisées aussi massivement. On peut regretter cette absence de données parce que ces substances sont de puissants perturbateurs endocriniens alors que les études épidémiologiques montrent une augmentation des altérations des fonctions de reproduction ou des dérégulations hormonales non seulement chez les travailleurs exposés mais aussi dans la population générale. Dans ce contexte, il serait très important de disposer de techniques de mesures permettant d'évaluer la totalité des substances présentes dans l'alimentation ou dans un vecteur environnemental (air ou eau), sans être obligé de mesurer par des techniques analytiques de plus en plus complexes chaque composé potentiellement impliqué. Dans ce but, de nouvelles méthodologies basées sur des bio-essais très spécifiques (par exemple gènes reporteurs) sont déjà disponibles sur le marché, parfaitement adaptées à l'approche de type TEFs.

Tableau 6. Evaluation des risques pour les autres PTS que les pesticides OC.

\begin{tabular}{|c|c|c|c|c|c|}
\hline Sources & Quantités & Toxicité & Vecteur & \% DJT & \\
\hline PAHs & $\begin{array}{l}\text { Produit de } \\
\text { pyrolyse Pollution } \\
\text { pétrolière }\end{array}$ & $\begin{array}{l}1000 \text { T/an Région } \\
\text { méd. }\end{array}$ & $\begin{array}{l}\text { Cancérigènes }(2 \mathrm{~A}, 2 \mathrm{~B}, 3) \\
\text { Perturbateurs } \\
\text { endocriniens }\end{array}$ & $\begin{array}{l}\text { Viandes } \\
\text { cuites } \\
(70 \%)\end{array}$ & $210-6$ \\
\hline
\end{tabular}




\begin{tabular}{|c|c|c|c|c|c|}
\hline Dioxines & $\begin{array}{l}\text { Contaminants des } \\
\text { O.C. } \\
\text { Sous-produits de } \\
\text { combustion } \\
\text { Traitement des } \\
\text { pâtes à papier }\end{array}$ & $\begin{array}{l}800 \mathrm{~g} / \mathrm{an} \quad \text { (France } \\
2000 \text { ) }\end{array}$ & $\begin{array}{l}\text { Cancérigène } \\
\text { hépatotoxique } \\
\text { Perturbateurs } \\
\text { endocriniens } \\
\text { Tératogène, } \\
\text { immunotoxique }\end{array}$ & $\begin{array}{l}\text { Produits } \\
\text { animaux } \\
(60 \%) \\
\text { produits } \\
\text { de la mer } \\
(28 \%)\end{array}$ & $\begin{array}{l}100- \\
160 \%\end{array}$ \\
\hline PCBs & $\begin{array}{l}\text { Huiles de } \\
\text { transformateurs } \\
\text { Fluides } \\
\text { caloporteurs et } \\
\text { hydroliques, } \\
\text { lubrifiants, } \\
\text { peintures, encres }\end{array}$ & $\begin{array}{l}55000 \mathrm{~T} \quad \text { (stock } \\
\text { France } 1987 \text { ) }\end{array}$ & $\begin{array}{l}\text { Cancérigène } \\
\text { hépatotoxique } \\
\text { Perturbateurs } \\
\text { endocriniens } \\
\text { Immunotoxique, } \\
\text { neurotoxique }\end{array}$ & $\begin{array}{l}\text { Produits } \\
\text { de la mer } \\
(70 \%)\end{array}$ & $190 \%$ \\
\hline BFRs & $\begin{array}{l}\text { Ignifugeants pour } \\
\text { électricité, } \\
\text { bâtiment, } \\
\text { transport, textile }\end{array}$ & $\begin{array}{l}30860 \mathrm{~T} \\
\text { (Consommation } \\
1999 \text { Europe) }\end{array}$ & $\begin{array}{l}\text { Cancérigènes probables, } \\
\text { Perturbateurs } \\
\text { endocriniens } \\
\text { Neurotoxiques }\end{array}$ & $\begin{array}{l}\text { Produits } \\
\text { de la mer } \\
(75 \%)\end{array}$ & $\begin{array}{l}(30 \% \\
\text { DJT } \\
\text { PCBs) }\end{array}$ \\
\hline Alkylphénols & $\begin{array}{l}\text { Détergeants, } \\
\text { produits } \\
\text { nettoyage, } \\
\text { agriculture, tissus, } \\
\text { peintures... }\end{array}$ & $\begin{array}{l}77600 \mathrm{~T} \\
\text { (consommation } \\
\text { Europe 1997) }\end{array}$ & Oestrogénomimétiques & & \\
\hline Phtalates & $\begin{array}{l}\text { Plastifiants } \\
\text { additifs encre, } \\
\text { peintures, } \\
\text { adhésifs, } \\
\text { lubrifiants, } \\
\text { cosmétiques, } \\
\text { solvants... }\end{array}$ & $\begin{array}{l}1 \text { million de } T \\
\text { Produits en } \\
\text { Europe de l'Ouest } \\
109 \text { KT France }\end{array}$ & $\begin{array}{l}\text { Perturbateurs } \\
\text { endocriniens } \\
\text { Cancérigène? }\end{array}$ & & \\
\hline
\end{tabular}

Pour les formes organiques des métaux lourds, nous disposons d'une étude récente de l'AFSSA sur le méthyl-mercure chez les forts consommateurs de poissons dans différentes catégories d'âge (des enfants de 3-8 ans aux adultes de plus de 65 ans). L'exposition maximale au $95^{\mathrm{e}}$ percentile est constatée pour les jeunes enfants ( $133 \%$ de la DHTP), l'exposition minimale est notée pour les adultes de 15 à 24 ans ( $50 \%$ de la DHTP). Une étude importante a été effectuée dans différents pays méditerranéens par le MEDPOL basée sur la teneur en mercure et méthylmercure dans les cheveux de pêcheurs grands consommateurs de poissons. Les résultats ont montré qu'aucune des populations étudiées ne présentait de concentrations correspondant à des niveaux de risques 
significatifs.

L'ensemble des données sur les vecteurs d'exposition aux PTS montre que les lipides sont les vecteurs majeurs de la contamination le long des chaînes alimentaires et que l'homme est exposé essentiellement via les lipides animaux (lait, poissons, œufs). La maîtrise des niveaux de PTS dans l'alimentation de I'homme et des animaux de rente est un maillon essentiel dans les stratégies de diminution d'exposition à ces substances.

\section{Conclusions et recommandations}

Le rapport du PNUE est une première tentative pour réaliser un bilan aussi exhaustif que possible aux niveaux régional et mondial sur les différents aspects des PTS, dépassant largement les précédents rapports se limitant aux POPs. La collection et le traitement des données ayant servi de base à la rédaction de ces rapports ont montré toute la difficulté d'un tel exercice en l'absence de structures organisées aux niveaux national, régional et mondial pour informer réellement les instances politiques et économiques sur l'état de contamination de notre planète. II est certain que l'usage inconsidéré de substances chimiques persistantes et de procédés industriels très polluants a amené une contamination généralisée de notre environnement, de notre alimentation et de notre corps. Les moyens investis pour la connaissance de ces pollutions et de leurs effets sur l'environnement et sur I'homme sont dérisoires par rapport à ceux consacrés au développement et à la promotion de l'usage de ces produits ou procédés. Il semble que l'expérience de l'usage industriel des produits chimiques ne nous ait pas servi de leçon (voir ce qui se passe avec les OGMs) et cette politique globale reste à construire.

Dans ce but, plusieurs recommandations ont été faites, que l'on peut résumer de la façon suivante : - Il existe de nombreux manques pour se faire une idée précise de la contamination des différents milieux, en particulier dans les sols, l'eau, les boues et les zones de stockage. De plus il y a un manque dramatique de données dans les pays du Sud qui utilisent largement les produits et les technologies issues de pays du Nord sans avoir les moyens réels d'en contrôler l'usage. Il est donc important de constituer des réseaux nationaux et internationaux d'analyses en étroite interaction et utilisant des méthodes standardisées dans le but de réaliser des inventaires performants.

- Au niveau écotoxicologique il manque des données sur les nouvelles familles chimiques incluses dans les PTS (alkyl phénols, PBDEs, phtalates...). Une attention toute particulière doit être portée à l'identification des réservoirs de contaminants, sources futures de pollution. De plus il est indispensable de développer de nouveaux outils pour estimer la globalité des composés présents dans un secteur et induisant le même type d'effet, par exemple des bio-essais basés sur l'affinité à certains récepteurs ou sur des réponses cellulaires spécifiques (AhR, ER, VTG...). Ici aussi doivent être développés et interconnectés des réseaux de surveillance utilisant des techniques de modélisation et d'évaluation standardisées.

- Au niveau des effets sur l'homme il semble que les réseaux de surveillance relatifs aux risques chimiques soient encore plus embryonnaires que dans le domaine de l'environnement. II s'agit de suivre les charges corporelles en PTS dans les populations en fonction de nombreux facteurs comme l'âge, le sexe, les habitudes alimentaires, la profession, le niveau d'éducation, les comportements particuliers (tabac, drogues, alcool). Ceci nécessite des dosages dans les tissus représentatifs comme les phanères, le lait et le sang, ces deux derniers étant vecteurs d'exposition pour le fœetus et le nouveau-né. De même, la surveillance épidémiologique doit être renforcée pour mieux apprécier les 
effets des faibles niveaux d'exposition et les effets des cocktails, en utilisant les biomarqueurs pertinents (adduits, activités enzymatiques, anticorps, sondes...) aujourd'hui disponibles mais encore limités au cadre de la recherche.

L'absence de mise en place d'une telle politique intégrée au niveau international posera de sérieux problèmes d'ordre scientifique, environnemental et politique au développement économique durable des populations méditerranéennes et plus généralement des habitants de notre planète.

\section{RÉFÉRENCES}

1. Regionally Based Assessment of Persistent Toxic Substances : Mediterranean Regional Report, Décembre 2002. UNEP Chemicals, 11-13 Chemin des Anémones, CH-1219 Châtelaine, Genève Suisse.

2. Regionally Based Assessment of Persistent Toxic Substances : Global Report 2003, UNEP Chemicals, 11-13 Chemin des Anémones, CH-1219 Châtelaine, Genève Suisse. n

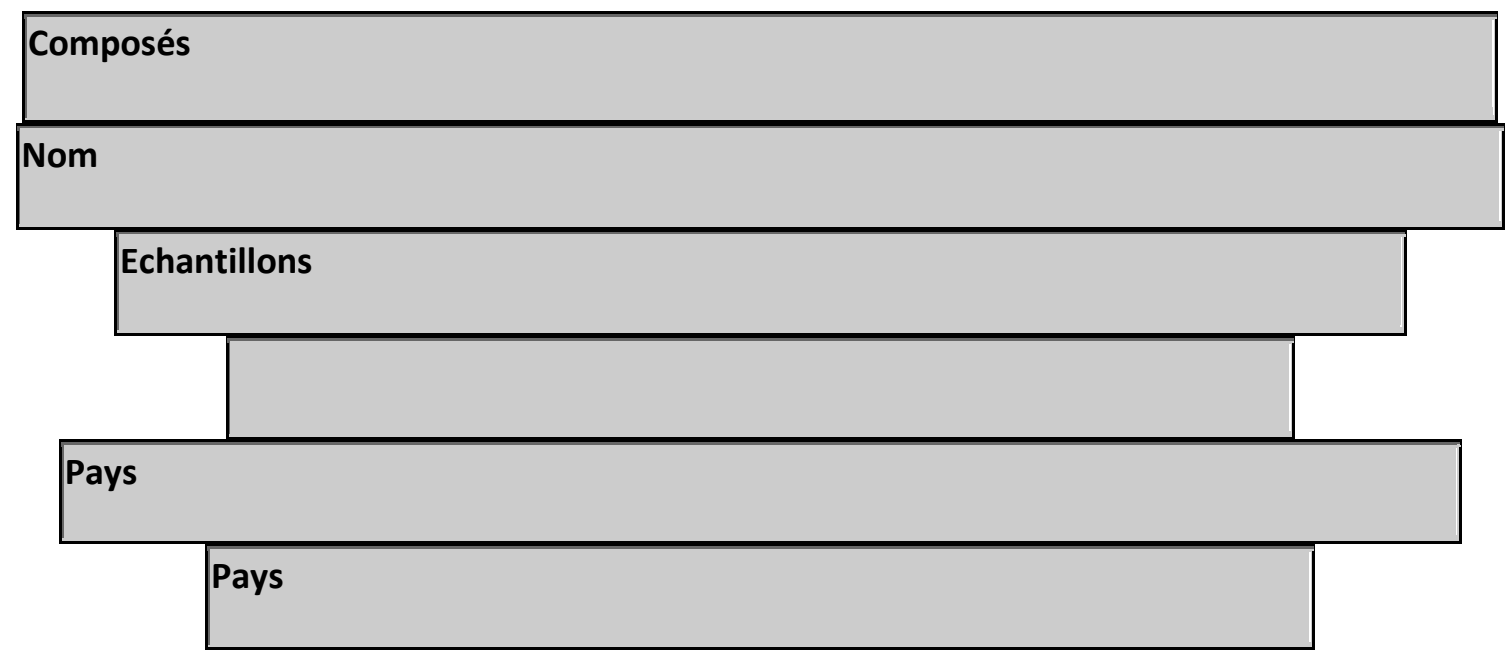




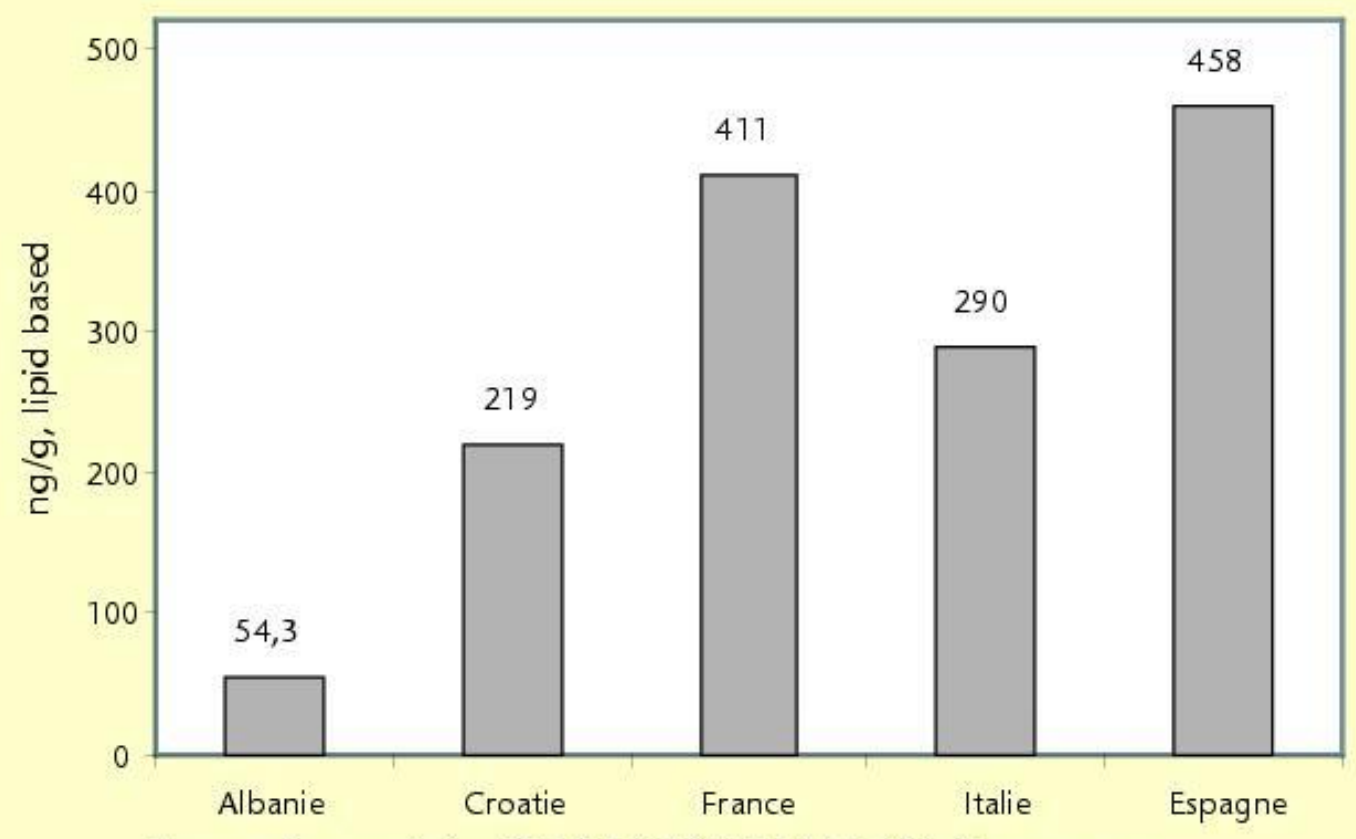

Somme des congénères $C B 28,52,101,138,153,180$ dits marqueurs

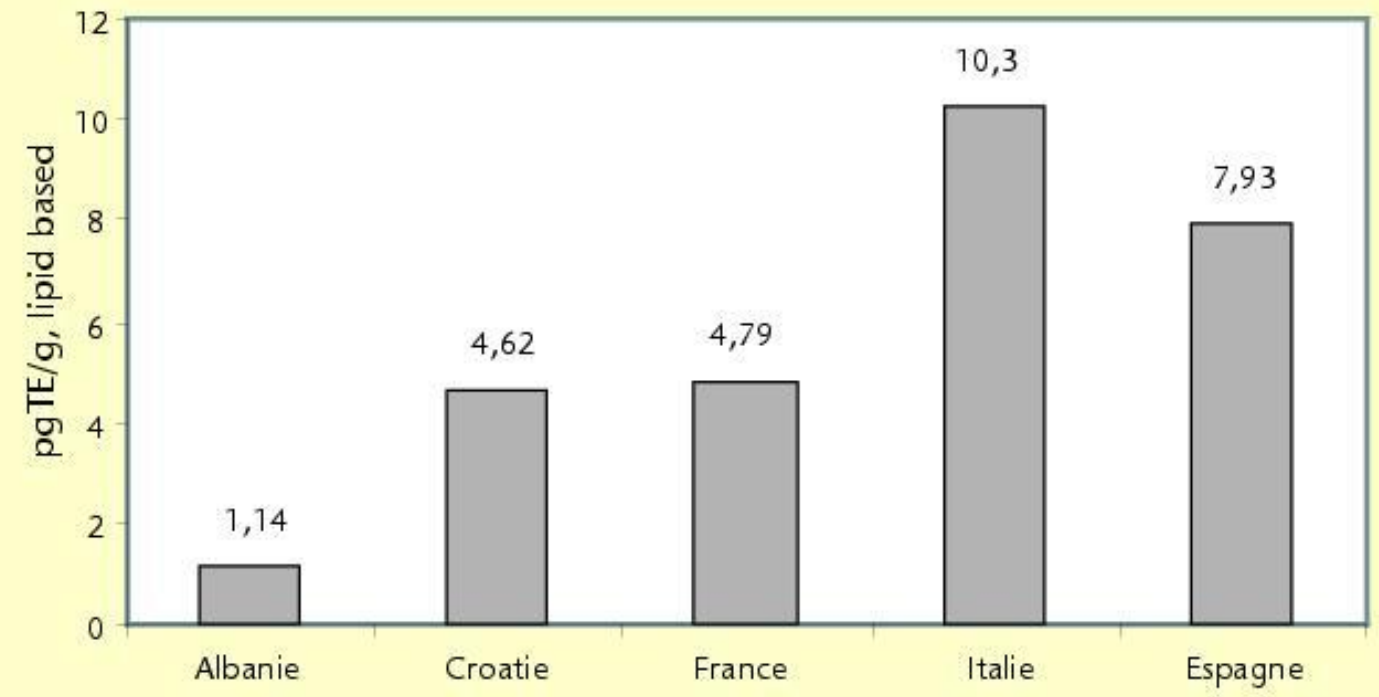

Somme des congénères $77,126,169$ dits dioxinelike

Figure 1. Teneurs en PCBs dans le lait humain mesurées dans différents pays (1993-1996). 


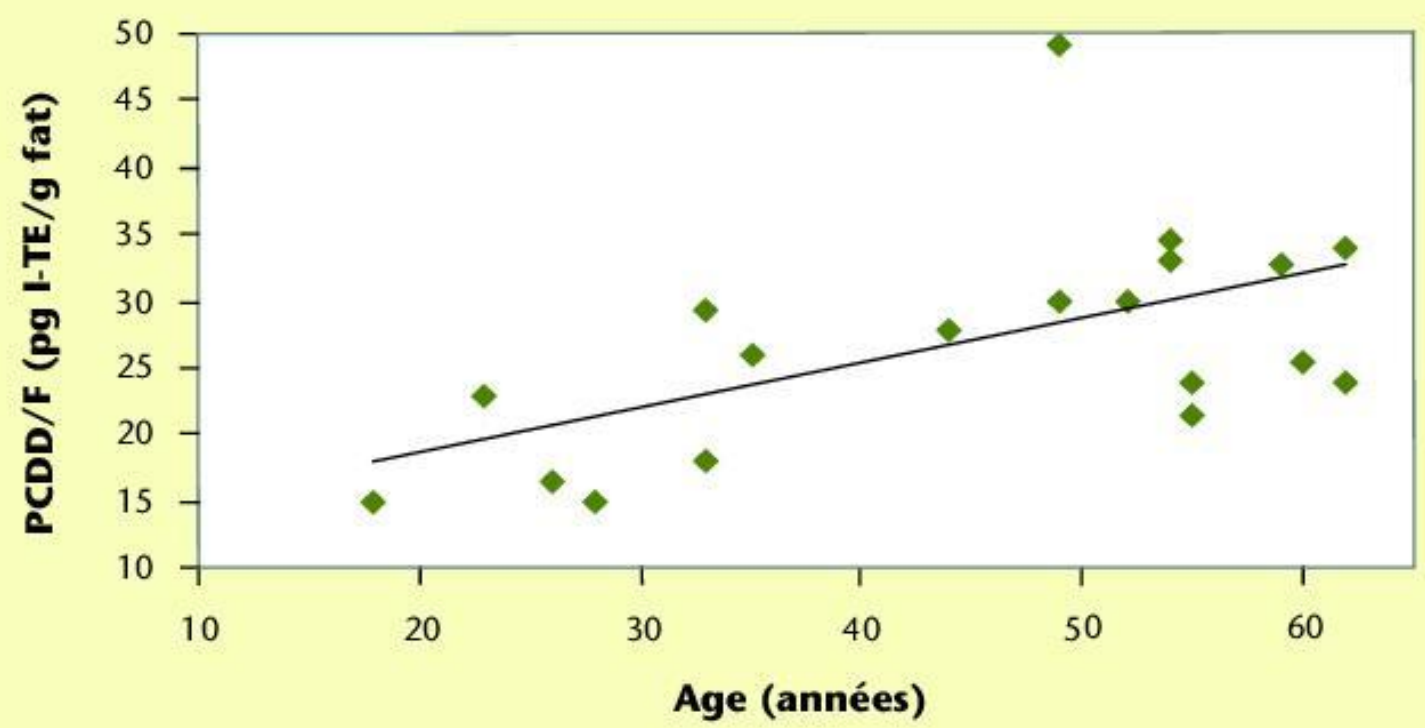

Figure 2. Relation entre âge et concentration en PCDDs and PCDFs dans le plasma. 

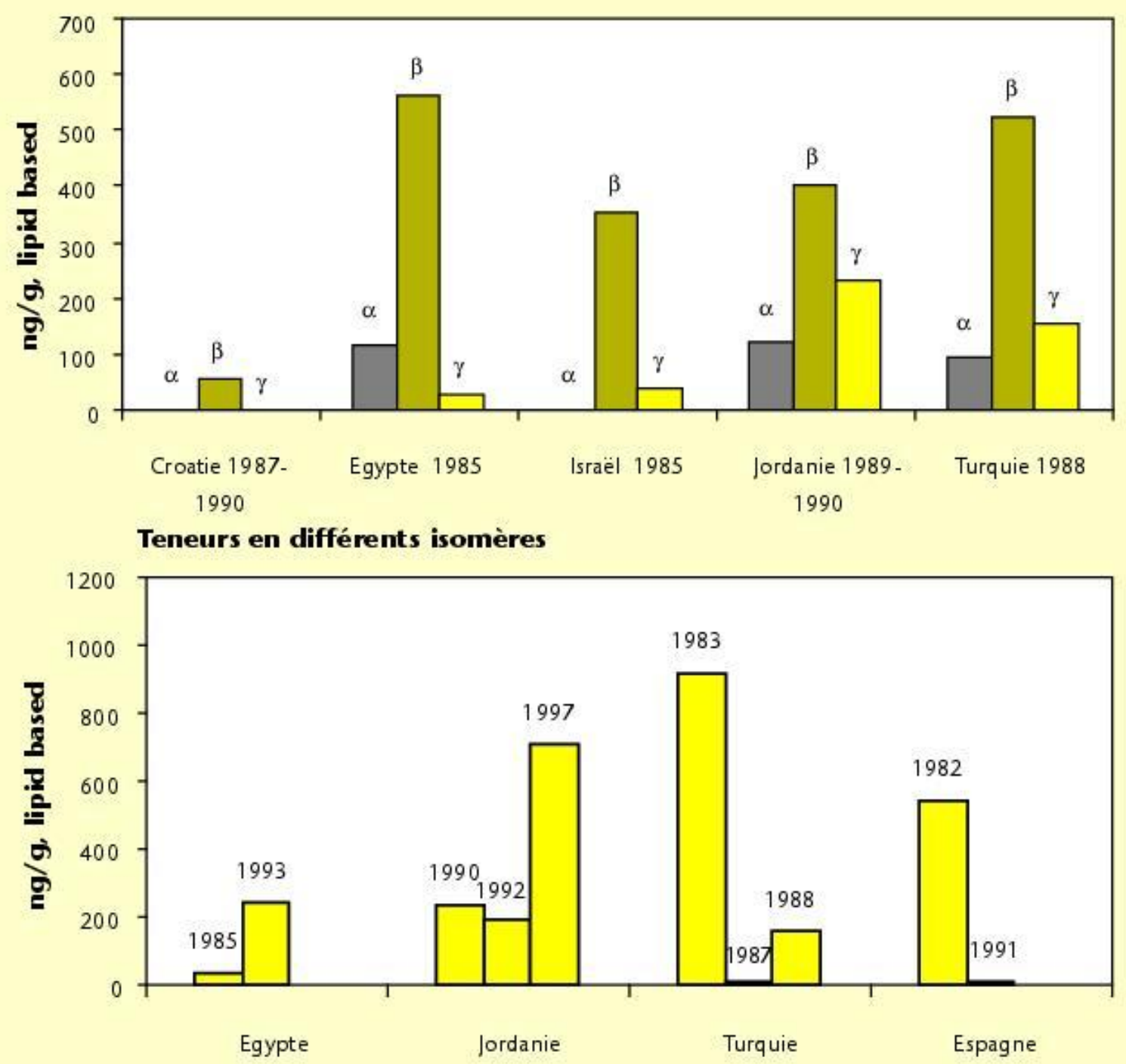

Evolution des teneurs en isomère $\gamma$ au cours de la période 1983-1997

Figure 3. Teneurs en $\mathrm{HCH}$ s dans le lait humain mesurées dans différents pays de la région. 


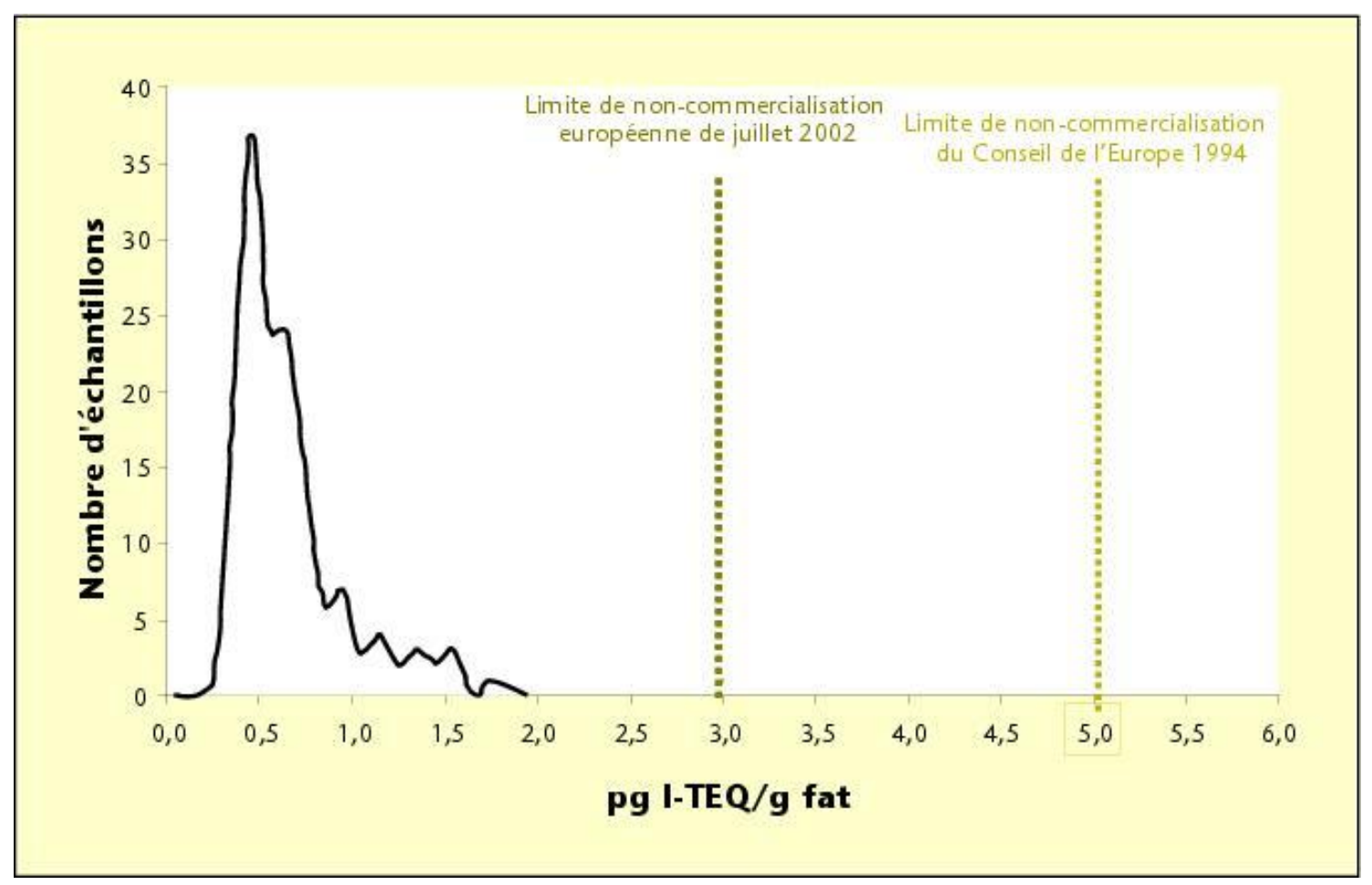

Figure 4. Distribution des teneurs en dioxines dans 148 échantillons de lait de grand mélange prélevés en France (1999). 